\title{
Apports de la modélisation numérique à l'étude de la dynamique hydro-sédimentaire de l'estuaire de la Rance
}

\section{Thierry Denot}

Electricité de France - Laboratoire National d'Hydraulique et

Environnement - 6 quai Watier - 78400 Chatou

Pierre Lang

SOGREAH - 6 rue de Lorraine - 38130 Echirolles

\section{Résumé}

Situé dans une zone où les marées sont très marquées, l'estuaire de la Rance a fait l'objet de plusieurs aménagements dont le plus remarquable est sans conteste son usine marémotrice. Depuis quelques années, les évolutions morphodynamiques de l'estuaire semblent s'être accélérées. Les Elus et Usagers de la Rance, regroupés au sein du comité CCEUR ont décidé de mettre en cuvre une modélisation hydro-sédimentaire de l'estuaire, réalisée par le groupement EDF-LNHE et SOGREAH. Trois modèles, basés sur le système TELEMAC développé par EDF-LNHE, ont donc été construits. Is sont analysés conjointement pour permettre une représentation fidèle de la dynamique hydrosédimentaire de l'estuaire.

\section{Abstract}

Located in an area characterized by strong tides, the Rance estuary has been influenced by many water works. No doubt the most famous is its tidal power plant. For a few years, morphodynamical evolutions seem to be stronger. The CCEUR committee, gathering elected people and users of the estuary, has decided to finance a hydrosedimental study of the estuary, performed by the group EDF-LNHE and SOGREAH. Three models, based on the TELEMAC system developed by EDF-LNHE, have been built. They are analysed together to aliow a complete overview of the hydrosedimental dynamics of the estuary.

\section{Introduction}

\subsection{Contexte et objectifs de l'étude}

L'estuaire de la Rance est unique en son genre. Situé dans une zone où les marées sont très marquées (près de $14 \mathrm{~m}$ d'amplitude en vive eau exceptionnelle à Saint-Malo), il a fait l'objet de plusieurs aménagements dont le plus remarquable est sans conteste son usine marémotrice. Le régime de marée dans l'estuaire est désormais fonction du régime d'exploitation de l'usine (simple effet, double effet, pompage, etc.). Globalement, ceci a conduit à une augmentation du niveau d'eau moyen dans l'estuaire et à un élargissement de la durée des étales. 
Depuis quelques années, les évolutions morphodynamiques de l'estuaire semblent s'être accélérées avec notamment un envasement assez net de la partie amont et des anses de l'estuaire. Les Elus et Usagers de la Rance, regroupés au sein du comité CCEUR ont décidé de mettre en cuvre une modélisation hydrosédimentaire de l'estuaire, réalisée par le groupement EDF-LNHE et SOGREAH.

\subsection{Déroulement}

L'étude a démarré début 1998. Une collecte et une analyse critique des données existantes et des études antérieures ont été effectuées, complétées ensuite par une campagne de mesures en nature mise en œuvre par la société Hydroexpert. De plus, SOGREAH a entrepris des essais sédimentologiques en canal pour caractériser plus précisément le comportement des vases de l'estuaire. Il était effectivement inảispensable d'acquérir les données d'entrée des modèles (bathymétrie, débits des pompes et dess vannes de l'usine, paramètres sédimentologiques) ainsi que les données de validation (mesures de courants, de matières en suspension, de qualité de l'eau) pour les modèles numériques.

\section{Les logiciels utilîsés}

\subsection{Le système TELEMAC}

Ce système de logiciels est développé par EDF-LNHE. Différents modules permettent de modéliser la dynamique des écoulements, bi- ou tridimensionnels, dans les milieux naturiels (côtes, estuaires, lacs et rivières) et le transport de substances par ces écoulements: polluants conservatifs ou dégradables, sables, vases, etc.

\subsection{TELEMAC-2D}

TELEMAC-2D, module de calcul hydrodynamique bidimensionnel, résout les équations de Saint Venant. Comme pour les autres modules du système TELEMAC, la technique des éléments finis permet une grande souplesse lors du maillage des domaines d'étude: fort raffinement dans les zones délicates et maillage plus lâche dans les zones de moindre intérêt (voir figures 1 et 3 ). Les éléments de calcul sont des mailles triangulaires.

\subsection{TELEMAC-3D / SEDI-3D}

TELEMAC-3D résout les équations de Navier-Stokes. Il peut prendre en compte plusieurs traceurs actifs (i.e. qui agissent sur la densité du fluide, comme la température, la salinité ou le sédiment). Un des intérêts de ce module pour l'étude qui nous intéresse est qu'il utilise le même maillage que TELEMAC-2D, en le dupliquant sur la verticale : les éléments de calcul sont donc des prismes. Le module sédimentologique SEDI-3D, couplé avec l'hydrodynamique, permet de 
modéliser le dépôt, la remise en suspension et le tassement du sédiment de type vaseux.

\subsection{SUBIEF-2D}

SUBIEF-2D permet d'étudier le transport de un ou plusieurs traceurs au sein d'un écoulement à surface libre. Ces traceurs peuvent être dilués ou en suspension dans l'eau. Dans ce dernier cas, SUBIEF peut calculer leur dépôt sur le fond et leur remise en suspension. SUBIEF utilise un fichier hydrodynamique provenant de TELEMAC-2D (hydrodynamique et transport sédimentaire sont découplés).

Les deux aspects sont utilisés dans cette étude: transport de polluants (avec définition éventuelle d'un temps de dégradation caractéristique) et évolution sédimentaire. Pour ce second aspect, on définit des paramètres sédimentaires (diamètre et vitesse de chute des particules, contraintes d'érosion et de dépôt) qui permettent ensuite, par l'utilisation du fichier, de modéliser les évolutions bathymétriques.

\section{Description des modèles numériques}

Trois modèles d'emprises différentes ont été construits sur la zone d'étude. Ces modèles complémentaires ont chacun leurs objectifs, décrits dans les paragraphes suivants.

\subsection{Modèle $2 \mathrm{D}$ côtier}

Ce premier modèle est limité par la côte depuis le Cap Fréhel à l'Ouest jusqu'à Granville au Nord-Est, en incluant la baie du Mont-St-Michel. La figure 1 piésente le maillage du domaine. Celui-ci compte 9896 éléments et 5316 noeuds, avec des tailles de mailles variant de quelques mètres (abords de l'usine) à plus d'un kilomètre dans le domaine maritime.

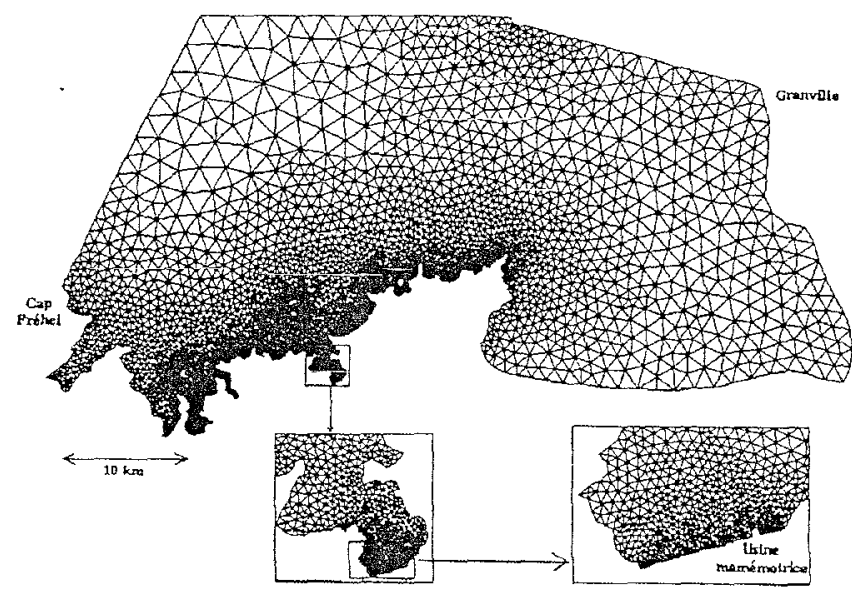

Figure 1: maillage du domaine côtier Figure 1: mesh of the coastal domain 
Ce modèle est utilisé principalement pour examiner la qualité des eaux dans la zone de débouché de la Rance. L'hydrodynamique a été calculée par TELEMAC$2 \mathrm{D}$ (validation, marées de coefficients caractéristiques), et SUBIEF est utilisé pour le modèle de qualité d'eau.

\subsection{Modèle 2D de l'estuaire}

Il couvre la partie de l'estuaire limitée par l'écluse du Châtelier à l'amont et l'usine marémotrice à l'aval. Il comporte 11566 mailles et 6281 noeuds. Les tailles de mailles sont comprises entre 5 et $150 \mathrm{~m}$. Ce modèle est complémentaire du précédent, dans la mesure où ils ne sont séparés que par le barrage de l'usine marémotrice (figures 2 et 3 ).

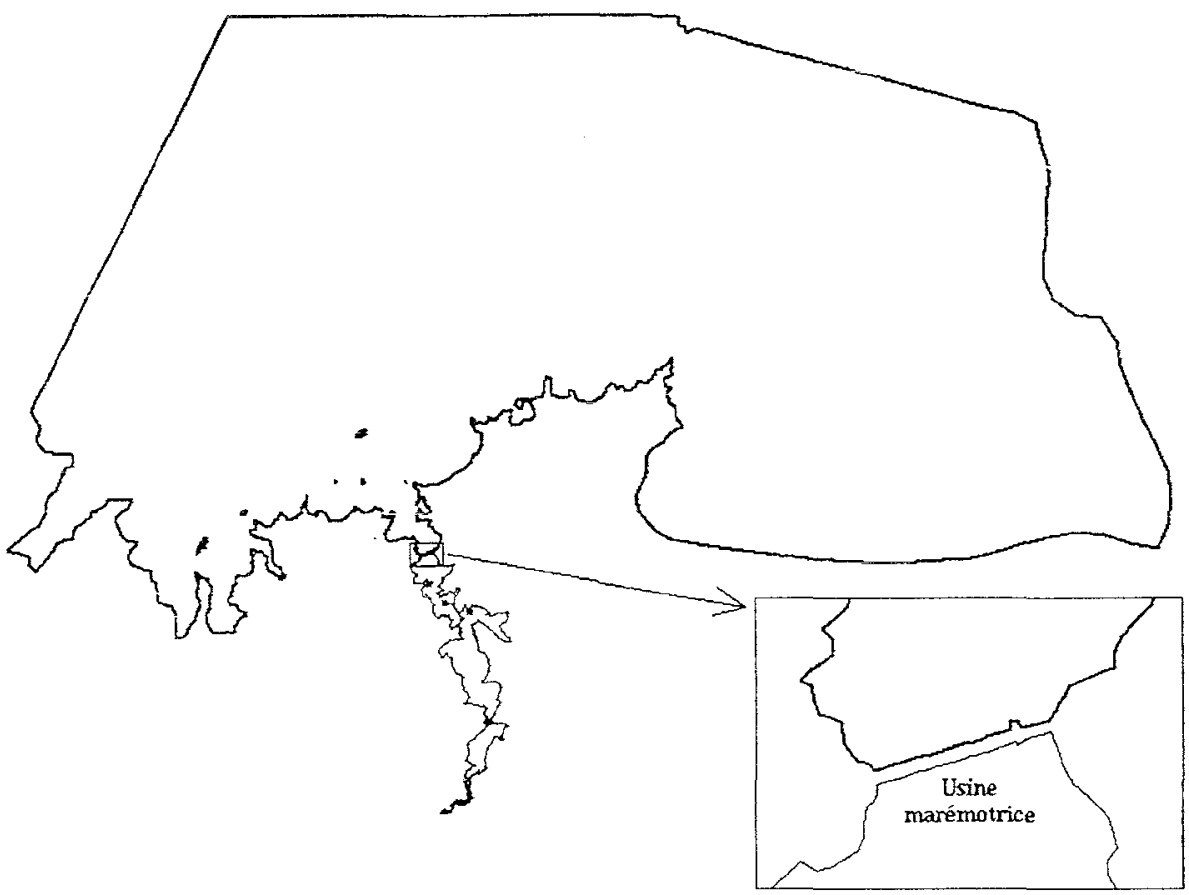

Figure 2 : limites des domaines côtier (trait gras) et estuarien (trait normal)

Figure 2 : limits of the coastal (bold) and estuarine (normal) domains

Deux types de problématiques sont étudiées sur le domaine estuarien.

La première est la qualité de l'eau: de très nombreux points de rejet (stations d'épuration, eaux usées) existent dans l'estuaire. L'optimisation de ceux-ci afin d'améliorer la qualité des eaux est une problématique importante.

La seconde, de loin la plus cruciale, conceme l'évolution sédimentologique dans l'estuaire. Comme précisé dans l'introduction, la tendance à l'envasement des anses et dè la partie amont semble s'être accélérée ces dernières années. Les buts de cette partie sont donc multiples: comprendre les mécanismes gouvernant l'évolution hydro-sédimentaire, dégager des tendances à long terme, et examiner différents scénarios de gestion de l'estuaire permettant d'influer sur cette évolution. 
Les deux modélisations réalisées sont bidimensionnelles: TELEMAC-2D calcule l'hydrodynamique et SUBIEF-2D la qualité de l'eau ( $1^{\text {er }}$ cas) ou les évolutions sédimentologiques ( $2^{\mathrm{e}}$ cas). A cette occasion, un modèle de tassement de la vase, identique à celui de SEDI-3D, a été inclus dans SUBIEF-2D.

\subsection{Modèle 3D de l'estuaire}

Ce modèle est inclus dans le précédent: il est limité par l'écluse du Châtelier à l'amont et le goulet Port St Hubert - Port St Jean à l'aval. Cette limite est reconnue comme le point où l'estuaire passe d'un caractère totalement maritime (du point de vue de la salinité notamment) à un caractère plus estuarien, avec une influence fluviale plus marquée. C'est dans cette partie que les effets de stratification, peu présents dans l'estuaire aval, commencent à apparaître, et que les turbidités sont les plus importantes.

Le logiciel utilisé sur cette partie du domaine est TELEMAC-3D et son module sédimentologique SEDI-3D. Tous les phénomènes physiques ayant lieu dans cette zone sont représentés: courantologie 3D, stratification saline et turbide, phénomènes d'érosion et de dépôt, module de tassement des dépôts. Le maillage utilisé est extrait du maillage décrit au paragraphe précédent (figure 3), qui est ensuite dupliqué sur la verticale. Seule une zone a dû être raffinée, le modèle 3D étant plus sensible aux gradients de bathymétrie. Le maillage de cette zone compte donc 2081 noeuds (ce qui donne par exemple 10405 noeuds si on choisit 5 plans sur la verticale).

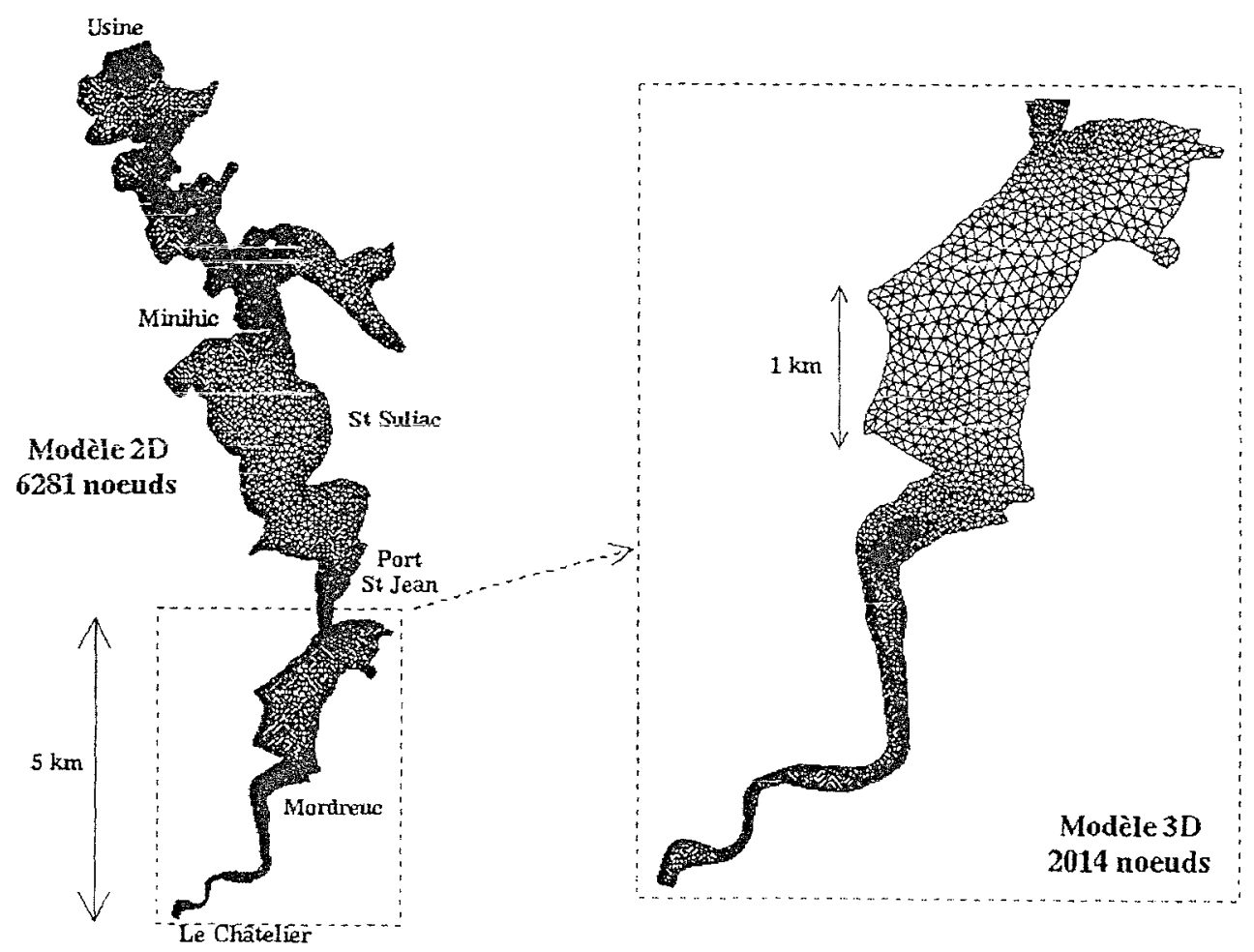

Figure 3 : maillages 2D et 3D de l'estuaire

Figure $3: 2 D$ and $3 D$ meshes of the estuary 


\section{Premiers résultats du système de modèles numériques}

\subsection{Modèle côtier}

La partie hydrodynamique a été validée par une simulation de la période du 9 au 11 novembre 1998, pour laquelle des mesures ont eu lieu. Par la suite, il a été exploité sur deux marées type (coefficients 45 et 95). Ces résultats sont maintenant exploités pour le modèle de qualité d'eau. Celui-ci est en cours de validation grâce à des mesures réalisées en 1998 par la société SAUNIERTECHNA (lâchers de rhodamine et de fluorescéine).

\subsection{Modèles hydrodynamiques ( $2 \mathrm{D}$ et $3 \mathrm{D})$ de l'estuaire}

Comme pour le modèle côtier, la validation a été effectuée sur la période du 9 au 11 novembre 1998. Durant cette période, le coefficient de marée pâsse de 73 à 44 , et le débit de la Rance est de $2 \mathrm{~m}^{3} / \mathrm{s}$. Les figures 4 et 5 présentent quelques comparaisons entre les mesures et les calculs ( $2 \mathrm{D}$ et le cas échéant 3D) en différents points de l'estuaire. Pour le modèle $3 D$, les résultats en vitesse et hauteurs d'eau du modèie $2 \mathrm{D}$ à Port $\mathrm{S}^{t}$ Jean ont été utilisés comme conditions aux limites. La figure 6 présente un exemple de courantologie 3D.

Les figures 5 et 6 montrent l'intérêt d'un modèle tridimensionnel : hydrodynamique mieux représentée et écoulements stratifiés dans certaines zones délicates.
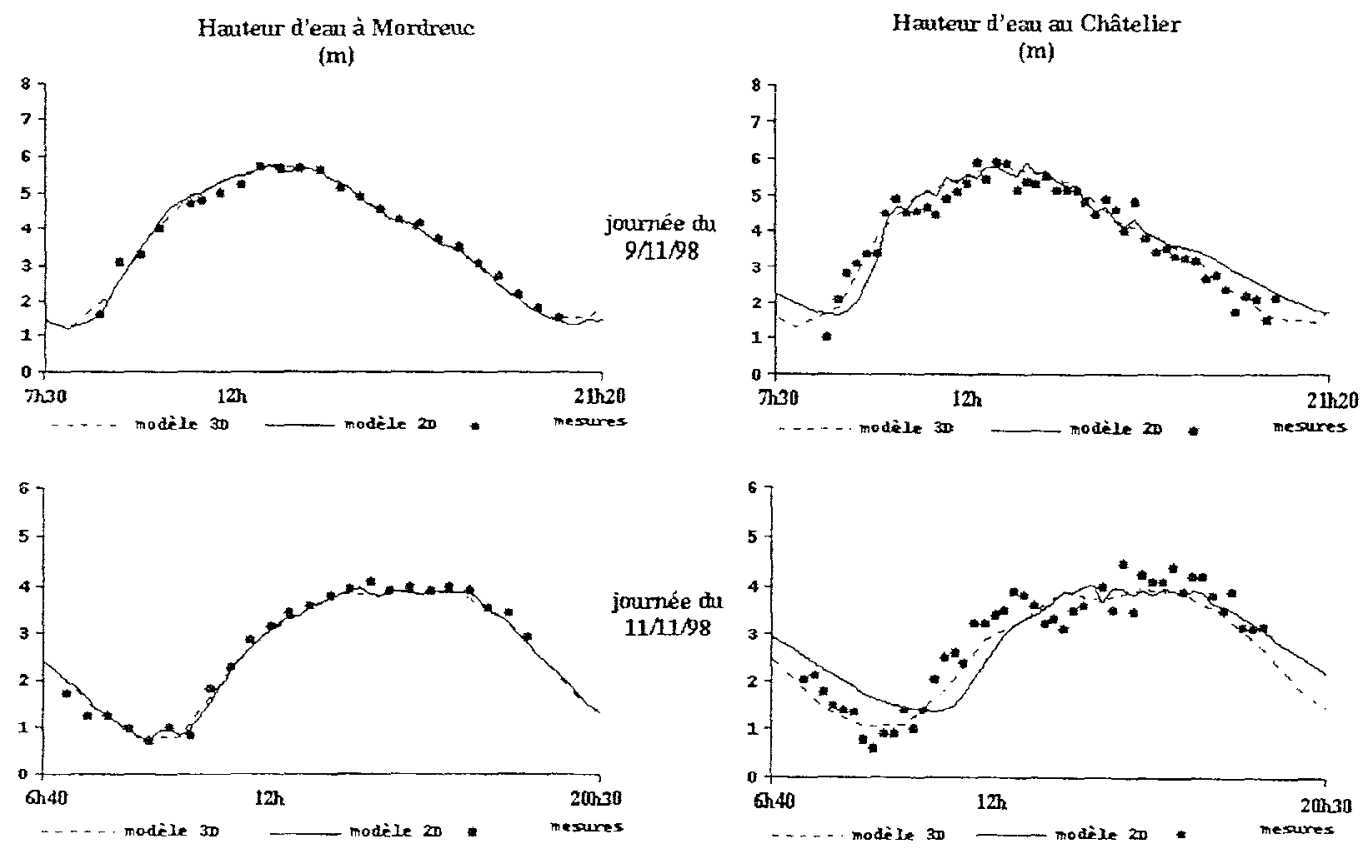

Figure 4 : Validation des modèles hydrodynamiques $2 \mathrm{D}$ et $3 \mathrm{D}$ Figure 4 : Validation of $2 D$ and $3 D$ hydrodynamic models 

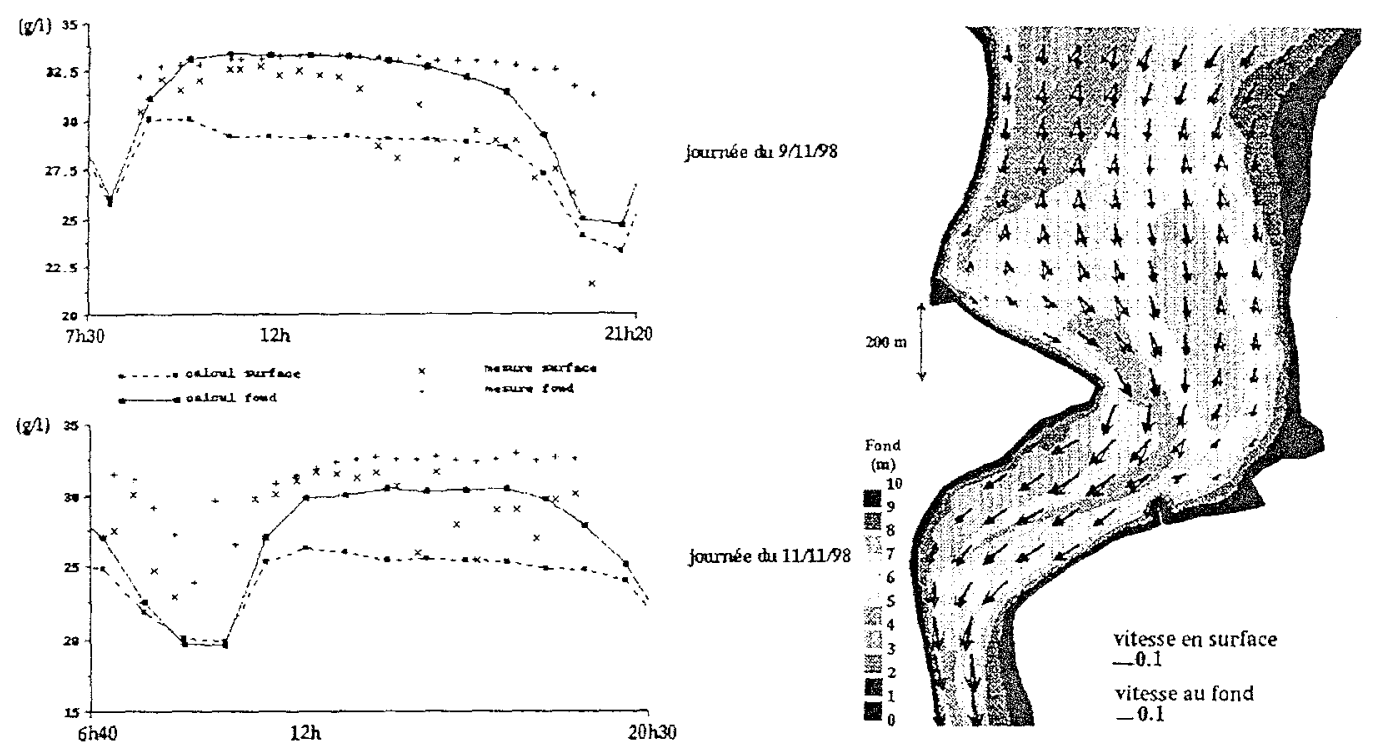

Figure 5: Validation en salinité du modèle 3D

Figure 6 : Courantologie 3D

Figure 5: Salinity validation of the $3 D$ model

Figure $6: 3 D$ hydrodynamics

\subsection{Modèle de qualité d'eau dans l'estuaire}

Ce modèle a été d'ores et déjà construit et est en cours de validation. Les 27 rejets les plus importants situés entre le Châtelier et l'usine (regroupés en 16 points) sont pris en compte.

Plusieurs simulations sont prévues avec deux types de polluants: les nitrates (polluant conservatif) et les colliformes fécaux qui ont un temps spécifique de disparition (T90: temps au bout duquel $90 \%$ des polluants ont disparu). Ces problèmes de quialité d'eau seront abordés en regroupant les modèles côtier ( $\mathrm{cf} \S$ 4.1) et estuarien, afin de simuler le transit des polluants à travers lé barrage.

\subsection{Modèles sédimentaires}

Ces modèles (2D et 3D) en sont au début de leur exploitation. Au vu des analyses faites, des mesures réalisées lors des campagnes [1] et des essais en laboratoire, et aussi des données d'études précédentes [2][3] l'ensemble des paramètres sédimentologiques a pu être fixé : vitesse de chute des sédiments, contraintes critiques d'érosion et de dépôt, vitesse de tassement de la vase.

Le paramètre le plus important concerne les conditions initiales. Il est en effet nécessaire d'imposer des épaisseurs de sédiment réalistes initialement, faute de quoi des dépôts «artificiels », provenant de la remise en suspension de sédiment qui n'a pas lieu d'être, pourraient se produire. Ces conditions seront déterminées en faisant tourner le modèle sur une longue période sans aucun dépôt initial, mais une eau très chargée en sédiment. La distinction sédiment tassé - couche remobilisable se fera d'elle-même, et c'est cette dernière que l'on choisira. 
Toute cette phase est d'une extrême importance, car la qualité des résultats sédimentologiques dépend du bon choix de ces paramètres.

\section{Perspectives}

Six calculs hydrodynamiques ont été réalisés, correspondant à deux débits de la Rance (crue et étiage) et trois types de coefficients (période de faible, de moyenne et de forte variation du coefficient de marée). Chacune de ces périodes est longue de 15 jours. Les évolutions sédimentologiques sur ces périodes seront ensuite combinées pour dégager des évolutions à long terme. Différents scénarios de gestion de l'estuaire (chasses au Châtelier par exemple) seront également examinés.

Dans cette étude, le modèle $2 \mathrm{D}$ sera utilisé préférentiellement. Le modèle 3D, nécessitant des temps de simulation plus longs, sera utilisé en appui et complément du précédent. İl permettra de préciser ou d'affiner les résultats de la partie amont, la plus critique en termes d'envasement.

\section{Références}

[1] LORIN J. (1999) : Modétisation hydraulique et sédimentaire du bassin de la Rance - Campagne de mesure en nature (1998), Rapport HYDROEXPERT, Fascicules 1 à 3 . *

[2] BONNOT-COURTOIS C., ET LAFOND L.R. (1991): Caractérisation et comportement des vases dans l'estuaire de la Rance, $113 \mathrm{p}$.

[3] L.C.H.F (1982) : Etude sédimentologique de l'estuaire de la Rance, Rapport général, $21 \mathrm{p}$.

* Rapport produit dans le cadre de l'étude 